\title{
Guest editorial to the special section on ECMFA and ICMT at STAF 2016
}

\author{
Modeling and model transformations research in 2016
}

\author{
Pieter Van Gorp ${ }^{1}$. Andrzej Wąsowski ${ }^{2}$
}

Received: 2 January 2018 / Accepted: 12 January 2018 / Published online: 6 February 2018

c) Springer-Verlag GmbH Germany, part of Springer Nature 2018

\section{Introduction}

Model-based engineering (MBE) is an approach to the design, analysis and development of software and systems that relies on exploiting high-level models and computerbased automation to achieve significant boosts in both productivity and quality. Model transformation (MT) is the field where engineers leverage different transformation paradigms (including rule-based transformations, term rewriting and manipulations of objects in general-purpose programming languages) to solve complex transformation problems. Research in these domains covers studies of algorithms, language and design aspects (modularity, composability and parameterization), tools and application domains.

The 12th European Conference on Modelling Foundations and Applications (ECMFA) and the 9th International Conference on Theory and Practice of Model Transformations (ICMT) were held as part of STAF 2016 in Vienna, Austria, from July 4, 2016, to July 5, 2016. As STAF events, both conferences focus on practical and foundational advances in software technology.

\section{Special section background and overview}

The objective of this special section is to provide a representative sample of advanced research emerging from ECMFA and STAF.

Both ECMFA and ICMT had rigorous reviewing processes. Each ICMT paper had already been reviewed by

\footnotetext{
Pieter Van Gorp

p.m.e.v.gorp@tue.nl

$凶$ Andrzej Wąsowski

wasowski@itu.dk

1 Eindhoven University of Technology, Eindhoven, The Netherlands

2 IT University of Copenhagen, Copenhagen, Denmark
}

at least four independent experts, and each ECMFA paper received at least three reviews. Conflicts of interest were prevented based on author and abstract screening. All special section reviewers were recruited from the original pool of reviewers for the conference papers. Additional reviewers have also been added for the extended versions. Each special section paper has undergone multiple improvement cycles with associated rebuttal letters. Ultimately, two ECMFA papers plus two ICMT papers were accepted from the initial pool of 66 ECMFA submissions and 36 ICMT submissions.

The selected four papers do cover a diverse set of topics (ranging from stress-testing API performance to the role of humans in the language design process) although all papers have a strong focus on tooling. In any case, MBE and MT tools should not be regarded as a non-scientific final end product. This is illustrated by the paper on MOMoT [1], in which that tool is used to generate new knowledge on the strengths and weaknesses of search algorithms in a transformation context. Strikingly, the related ICMT 2016 contribution was "just" a tool demonstration, but the work received such positive reviews and discussions that the potential for an extended version in this special section was clear. The authors have also seized the opportunity to generate new experiment-based insights with regard to local versus global search settings.

The paper on transformation clone detection had received the best paper award at ICMT [4]. Besides the strong study design, the paper excels in its novelty. While clone detection had already been studies in the broader MBE setting, it was previously unexplored in the MT context. As for all papers in this special section, the authors also benefited from highquality feedback from reviewers (first at the conference level and second at the level of the special section extensions).

The work on remote model querying [2] focuses on the storage and access technology for models. With models becoming very large, local access becomes both costly (it is too slow to copy entire models) and impractical (many designers need simultaneous access to large models). Thus modeling tools begin to face similar problems as 
database systems. The paper evaluates effectiveness of several approaches to querying remote models, showing that the design of the query API has bigger impact on efficiency than the network protocols used. This shows that scalability issues should be addressed by modeling-specific storage and access technologies.

The last paper [3] is concerned with supporting humans in creating meta-models, so models that describe the scope of modeling languages. It is widely recognized that examples of concrete models constitute a very useful input for designers creating meta-models. This paper attempts to support the role of humans in the language design process with tools that create meta-models semiautomatically, based on the provided example instances.

Summarizing, this special section includes the following four papers:

- A local and global tour on MOMoT [1], based on ICMT,

- Model clone detection for rule-based model transformation languages [4], based on ICMT,

- Stress-testing remote model querying APIs for relational and graph-based stores [2], based on ECMFA,

- An example is worth a thousand words: Creating graphical modeling environments by example [3], based on ECMFA.

We hope you enjoy reading these papers and encourage you to submit also to future editions of ECMFA and ICMT at STAF. Their calls will be announced via software engineering mailing lists, the conference Web sites (successors of http://is. ieis.tue.nl/research/ICMT16/ and http://ecmfa2016.itu.dk/) and social media accounts (successors of https://twitter.com/ icmt2016 and https://twitter.com/staf2016).

Acknowledgements We would like to thank the various STAF 2016 committees for providing the opportunity to organize ECMFA and ICMT 2016, and especially to the general chair and the organization chair (Gerti Kappel and Tanja Mayerhofer). Many thanks to all those who submitted papers to both conferences and also to the reviewers who provided both constructive and critical comments. Finally, we would like to thank Martin Schindler from the SoSyM office, for his excellent support throughout the editorial process.

\section{References}

1. Bill, R., Fleck, M., Troya, J., Mayerhofer, T., Wimmer, M.: A local and global tour on MOMoT. Softw. Syst. Model. (2017). https://doi. org/10.1007/s10270-017-0644-3

2. Garcia-Dominguez, A., Barmpis, K., Kolovos, D.S., Wei, R., Paige, R.F.: Stress-testing remote model querying apis for relational and graph-based stores. Softw. Syst. Model. (2017). https://doi.org/10. 1007/s10270-017-0606-9

3. López-Fernández, J.J., Garmendia, A., Guerra, E., de Lara, J.: An example is worth a thousand words: Creating graphical modelling environments by example. Softw. Syst. Model. (2017). https://doi. org/10.1007/s10270-017-0632-7

4. Strüber, D., Acreţoaie, V., Plöger, J.: Model clone detection for rulebased model transformation languages. Softw. Syst. Model. (2017). https://doi.org/10.1007/s10270-017-0625-6

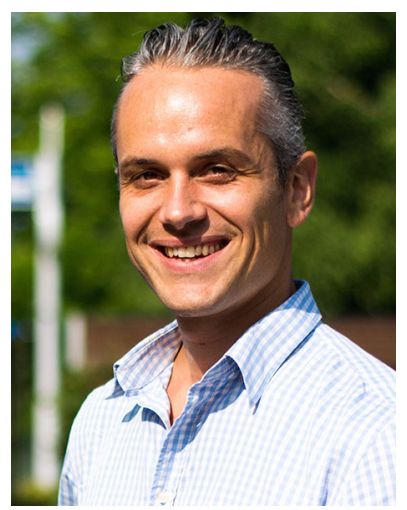

Pieter Van Gorp has obtained his $\mathrm{Ph} . \mathrm{D}$. degree in Software Engineering at the University of Antwerp. Since 2008, he has been an assistant professor in Information Systems at Eindhoven University of Technology. His research focuses on platform support for personal health data. In 2016, he has launched GameBus, a data-driven platform that rewards you for performing healthy activities in a social manner. He had previously designed an execution platform for securely analysing Personal Health Records in the cloud. Van Gorp is also known for his work on reproducible research and model transformations for UML, BPMN and Petri-Nets. Since late 2017, Van Gorp is managing the health-related research programmes of the Data Science Center Eindhoven while also performing research at Utrecht University of Applied Sciences.

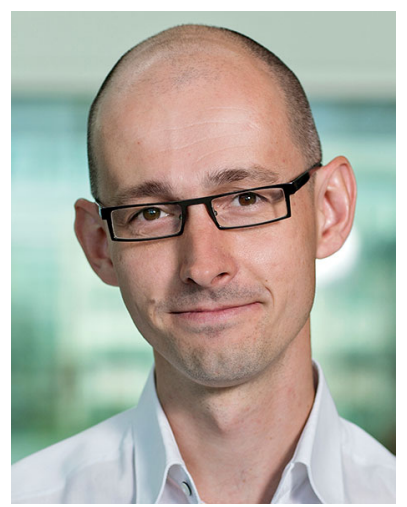

Andrzej Wąsowski is Professor of Software Engineering at the IT University of Copenhagen. Earlier, he worked also at Aalborg University in Denmark, and as visiting professor at INRIA Rennes and University of Waterloo, Ontario. His interests are in semantic foundations and tool support for model-driven development, especially for software product lines and componentbased systems. Many of these projects involve commercial or open-source partners, primarily in the domain of safety-critical embedded systems. Wassowski holds holds a PhD degree from the IT University of Copenhagen, Denmark (2005) and a MSC Eng degree from the Warsaw University of Technology, Poland (2000). He is a recipient of the Sapere Aude research leadership award from The Danish Council for Independent Research (2012). 